\title{
Correlative Fluorescence and Electron Microscopy of Graphene-Enclosed Whole Cells for High Resolution Analysis of Cellular Proteins
}

\author{
$\underline{\text { Indra Navina Dahmke }}^{1}$, Andreas Verch ${ }^{1}$, Diana B. Peckys ${ }^{2}$, Robert Weatherup ${ }^{3}$, Stephan Hofmann ${ }^{4}$ \\ and Niels de Jonge $e^{1,5}$
}

${ }^{1 .}$ INM - Leibniz Institute for New Materials, Saarbrücken, Germany.

2. Department of Biophysics, Saarland University, D-66421 Homburg, Germany.

3. Department of Chemistry, University of Cambridge, Cambridge, UK.

4. Department of Engineering, University of Cambridge, Cambridge, UK.

${ }^{5 .}$ Department of Physics, Saarland University, Saarbrücken, Germany.

Membrane proteins are the main targets of medical treatments, but their expression can vary in between different cells as well as between different areas within a cell [1]. This heterogeneity represents a major obstacle in tumor therapy since it can give rise to resistant tumor cells [2]. Yet, techniques to analyze the distribution of membrane proteins in their native environment remain limited [3]. Electron microscopy (EM) provides the necessary resolution but during conventional sample preparation, the information about the spatial arrangement within the context of the whole cell is usually lost and data is typically obtained from a few cellular regions only. Liquid-phase EM offers the opportunity to study series of whole hydrated cells but requires special experimental set-ups [4].

Here, we used bilayer graphene sheets to enclose hydrated breast cancer cells grown on silicon nitride $(\mathrm{SiN})$ and investigated the distribution of epidermal growth factor receptor ErbB2 in different cellular regions with correlative fluorescence microscopy (FM) and high-resolution EM (Fig.1).

SKBR3 breast cancer cells were seeded on 50-nm thick SiN windows supported by silicon microchips, and ErbB2 membrane proteins were specifically labeled with Quantum Dots (QDs) with a $\mathrm{CdSe}$ core emitting a red fluorescence. Then cells were then fixed and subsequently covered by a graphene sheet and examined with correlative FM and scanning transmission electron microscopy (STEM).

With STEM we achieved a spatial resolution of $2 \mathrm{~nm}$ that allowed for the identification of single, paired and clustered QDs (Fig. 2). Next, we performed statistical analyses and found varying numbers of functionally active homodimers in different cellular regions [5]. Herein, we focused on longdistance cell-cell connections named tunneling nanotubes, which also facilitate chemo-resistance in cancer cells [6]. We calculated the pair correlation function $g(r)$ of the radial pair distance $r$ between all label pairs in the images. Signaling active homodimers $(r=20 \mathrm{~nm})$ were predominantly found on the connecting site 1 (CON1) of the TNT (Fig. 2 a), whereas they were absent on the main part of the TNT body (TNT-) and CON2 implying that ErbB2 plays a role in TNT formation in SKBR3 cells.

In conclusion, the graphene enclosure allows for correlative light and electron microscopy of specifically labeled proteins in intact, hydrated cells. This capability was used for the inter- and intracellular analysis of the spatial arrangement of membrane proteins. Moreover, this is a versatile approach to study the immediate interaction of cancer therapeutics with their targets at the single-molecule level in order to elucidate potential causes of chemoresistance in heterogeneous cancer cell populations [7].

References:

[1] Y Arinaminpathy et al, Drug Discovery Today 14 (2009), p. 1130.

[2] RA Burrell et al, Mol Oncol 8 (2014), p. 1095. 
[3] X Chen et al, Nat Biotechnol 34 (2016), p. 1111.

[4] D Peckys et al, Sci Adv 1 (2015), e1500165.

[5] IN Dahmke et al, ACS Nano 11 (2017), p. 11108.

[6] E Lou et al, Comm Integr Biol 5 (2012), p. 399.

[7] We thank J Hermannsdörfer, U Korf, and E Arzt. Research was supported by the Leibniz Competition 2014. R.S.W. received a Research Fellowship from St. John's College, Cambridge \& a Marie Skłodowska-Curie Fellowship (Grant ARTIST no. 656870).

Cultivation of cells on microchip

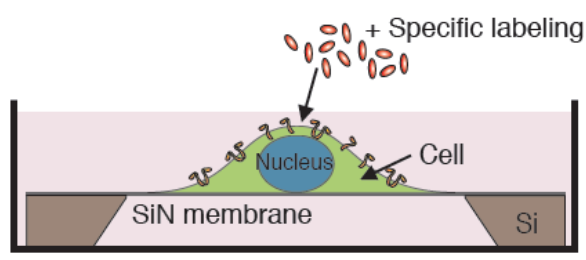

$\mathbb{R}=$ Transmembrane protein

\section{Examination with STEM}

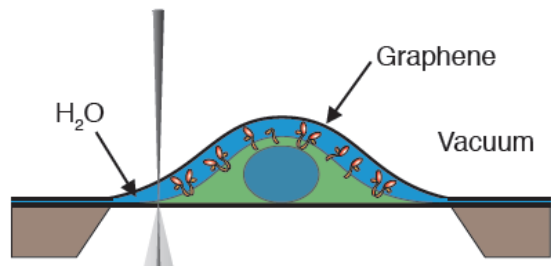

Electron beam

Figure 1. Schematic of the experimental approach. SKBR3 breast cancer cells were seeded on silicon microchips and membrane proteins (orange) were specifically labeled with Quantum Dots (QDs, red) Cells were then fixed, covered by a graphene sheet and examined with scanning electron microscopy (STEM). Figure 1 is reprinted from [5] with permission, copyright 2017 ACS Publications.

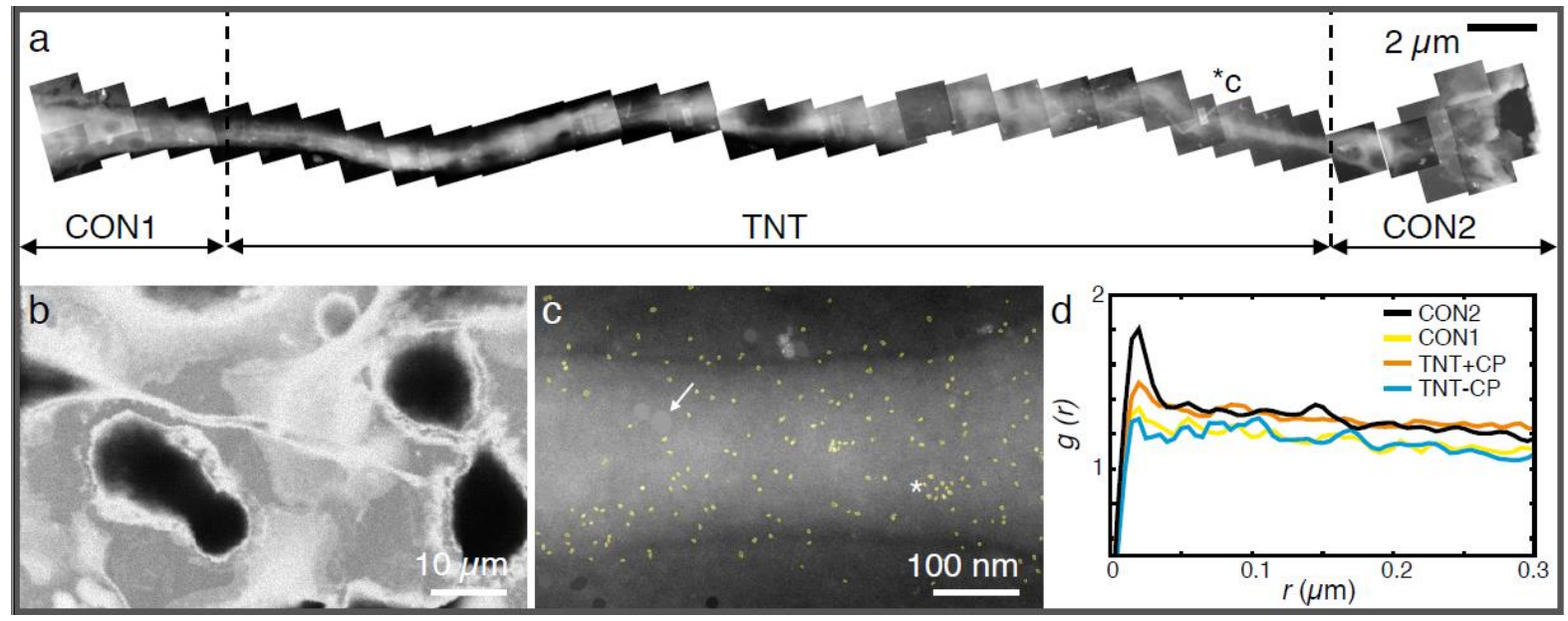

Figure 2. STEM images of a tunneling nanotube $(T N T)$. a) Stitched STEM images $(120,000 \times)$ show the distribution of QD-labeled ErbB2 proteins. Dashed lines mark the borders between the connection areas with the cells $(\mathrm{CON} 1,2)$ and the TNT. (b) Overview bright field STEM image. (c) Cropped image of the TNT marked with $*_{c}$ in a. ErbB2 proteins were labeled in yellow (* cluster). $\mathrm{Cu}$ contaminations $(\sim 50 \mathrm{~nm})$ were manually greyed out (arrow). (d) Pair correlation function $g(r)$ of radial pair distance $r$ between two labels for groups CON1, CON2 and TNT with (TNT+) and without (TNT-) additional cell membrane. Figure 2 is reprinted from [5] with permission, copyright 2017 ACS Publications. 